\title{
All-carbon-nanotube flexible multi-electrode array for neuronal recording and stimulation
}

\author{
Moshe David-Pur • Lilach Bareket-Keren • Giora Beit-Yaakov • \\ Dorit Raz-Prag • Yael Hanein
}

Published online: 24 August 2013

(C) The Author(s) 2013. This article is published with open access at Springerlink.com

\begin{abstract}
Neuro-prosthetic devices aim to restore impaired function through artificial stimulation of the nervous system. A lingering technological bottleneck in this field is the realization of soft, micron sized electrodes capable of injecting enough charge to evoke localized neuronal activity without causing neither electrode nor tissue damage. Direct stimulation with micro electrodes will offer the high efficacy needed in applications such as cochlear and retinal implants. Here we present a new flexible neuronal micro electrode device, based entirely on carbon nanotube technology, where both the conducting traces and the stimulating electrodes consist of conducting carbon nanotube films embedded in a polymeric support. The use of carbon nanotubes bestows the electrodes flexibility and excellent electrochemical properties. As opposed to contemporary flexible neuronal electrodes, the technology presented here is both robust and the resulting stimulating electrodes are nearly purely capacitive. Recording and stimulation tests with chick retinas were used to validate the advantageous properties of the electrodes and demonstrate their suitability for high-efficacy neuronal stimulation applications.
\end{abstract}

Moshe David-Pur and Lilach Bareket-Keren contributed equally to this work.

Electronic supplementary material The online version of this article (doi:10.1007/s10544-013-9804-6) contains supplementary material, which is available to authorized users.

M. David-Pur · L. Bareket-Keren · G. Beit-Yaakov · Y. Hanein

School of Electrical Engineering, Tel-Aviv University,

Tel-Aviv 6997801, Israel

M. David-Pur · L. Bareket-Keren · G. Beit-Yaakov • D. Raz-Prag • Y. Hanein $(\bowtie)$

Tel-Aviv University Center for Nanoscience and Nanotechnology, Tel-Aviv University, Tel-Aviv 6997801, Israel

e-mail: YaelHa@tauex.tau.ac.il
Keywords Carbon nanotubes · Multi electrode array · Neuronal recording $\cdot$ Neuronal stimulation $\cdot$ Flexible . Prosthesis

\section{Introduction}

Flexible neuronal micro electrode technology progressed extensively over the past several decades hand in hand with the overall development in the field of neuro-prosthetics. Several novel fabrication approaches suited for micro electrode applications were devised. These schemes attempt to achieve flexible electronic technology integration with high surface roughness while maintaining bio-compatibility and durability in physiological conditions. Commonly, these devices use metal electrodes such as gold (Sandison et al. 2002; Chen et al. 2009, 2011a; Wester et al. 2009; Lacour et al. 2010; Wei et al. 2011), titanium (Takeuchi et al. 2004), electroplated platinum black (Adams et al. 2005; Rodger et al. 2008; Graudejus et al. 2009, 2012; Rui et al. 2011), tungsten (Wei et al. 2011), platinum (Cheung et al. 2007; Mercanzini et al. 2008; Myllymaa et al. 2009; Viventi et al. 2011) and iridium (Rodger et al. 2008; Fomani and Mansour 2011) deposited on various flexible supports such as polyimide (Sandison et al. 2002; Takeuchi et al. 2004; Cheung et al. 2007; Viventi et al. 2011), parylene C (Rodger et al. 2008; Wester et al. 2009) or poly(dimethylsiloxane) (PDMS) (Graudejus et al. 2009, 2012; Lacour et al. 2010; Wei et al. 2011). These metal electrodes achieve neural stimulation by Faradaic current injection through the electrode-electrolyte interface. Electron transfer, associated with the Faradaic charge stimulation, can induce irreversible reduction and oxidation reactions that can damage both the electrode and the tissue (Merrill et al. 2005; Cogan 2008). Storage and injection of charge can also occur from valence changes in multivalent electrode coatings such as 
Iridium oxide (Robblee et al. 1983; Klein et al. 1989) that undergo reversible reduction-oxidation reactions (Merrill et al. 2005; Cogan 2008). Consequently, capacitive charge stimulation is preferable for neuronal stimulation, as it involves only a displacement current associated with charging and discharging of the electrode-electrolyte double layer (Merrill et al. 2005). Common capacitive electrode materials include titanium nitride (TiN), tantalumtantalum oxide and the more recently investigated carbon nanotubes (CNTs) (Rose et al. 1985; Gabay et al. 2007; Cogan 2008). Conducting polymers, such as polypyrrole (PPy) and poly(ethylenedioxythiophene) (PEDOT) are mixed conductors, exhibiting both electron and ion transport within the polymer film (Ludwig et al. 2006; Abidian et al. 2010; Blau et al. 2011).

A related key requirement in neuronal electrode technology is large specific capacitance $\left(\mathrm{C}_{\mathrm{s}}\right)$. Large specific capacitance reduces the electrode impedance, without increasing its geometric area. The reduction in impedance is essential for efficient, high resolution neuronal recording and stimulation (Robinson 1968; Loeb et al. 1995; Merrill et al. 2005; Cogan 2008). One of the best materials to exhibit both large specific capacitance as well as non-Faradaic behavior is porous TiN with $\mathrm{C}_{\mathrm{s}}$ in the range of $2 \mathrm{mFcm}^{-2}$ (Gabay et al. 2007). It was recently demonstrated that pristine CNTs exhibit similar performances to those of $\mathrm{TiN}$ with $\mathrm{C}_{\mathrm{s}}$ values in the range of $3-10 \mathrm{mFcm}^{-2}$ (Gabay et al. 2007). Accordingly, CNTs have been suggested by several studies as a future material for neuronal stimulation applications and several fabrication schemes have been studied. Primarily, direct growth of CNT electrodes (Wang et al. 2006; Gabay et al. 2007; Su et al. 2010) as well as CNT coatings of metal electrodes by electro-polymerization (Keefer et al. 2008), drop coating from a solution (Gabriel et al. 2009) and micro-contact printing (Fuchsberger et al. 2011) on a rigid support were described. To accommodate flexibility, CNT transfer onto a polymeric support (Su, Lin et al. 2009; Tsai et al. 2009; Carnahan et al. 2010; Chang-Jian et al. 2010) was recently presented. However, the lack of a simple platform to allow the realization of fully functional devices consisting of pristine CNT surfaces has left this technology so far largely unused.

Here, we present a novel flexible neuronal micro electrode device, based solely on multi-walled CNT (MWCNT) films embedded in a flexible polymeric support. We demonstrate a new simple and robust fabrication technique to realize the seamless CNT circuit on the flexible substrate. Next, the electrical and electrochemical properties of the CNT electrodes and of the CNT conducting traces were studied, using a scheme of specially designed electrode arrays. Finally, the flexible CNT MEA was applied for extracellular neuronal recording and stimulation of chick retinas.

\section{Methods}

\subsection{Flexible CNT MEA fabrication}

Flexible CNT MEAs were fabricated as follows. First, standard lithography (AZ1518 photoresist; Clariant) was used to form the desired circuit pattern on a Silicon/Silicon dioxide (Si/ $\mathrm{SiO}_{2}$ ) support. A $2.5 \mathrm{~nm} \mathrm{Ni}$ catalyst layer was deposited using an e-beam evaporator (VST). A resist lift-off process was then performed, followed by an oxygen plasma treatment to remove all photoresist residues. Next, MWCNTs were grown by chemical vapor deposition (CVD) (Lindberg Blue) with ethylene $(20 \mathrm{sccm})$ and hydrogen $(1,000 \mathrm{sccm})$ at $900{ }^{\circ} \mathrm{C}$. A flexible substrate, medical adhesive tape, parylene $\mathrm{C}$, polyimide or poly(dimethylsiloxane) (PDMS), was applied and peeled off with the CNT pattern. Medical adhesive tape (Steri-Drape, $3 \mathrm{M})$ was attached to the CNT pattern and pressed lightly. Parylene $\mathrm{C}$ was applied by on the CNT pattern by vapor deposition. Polyimide, prepared from a poly(pyromellitic dianhydride-co-4,4'-oxydianiline) 15 wt.\% solution in $\mathrm{N}$ methyl-2-Pyrrolidone (Sigma-Aldrich) was spin coated and cured at $350{ }^{\circ} \mathrm{C}$ under nitrogen atmosphere. Uncured PDMS (Sylgard 184, Dow Corning), mixed in a 10:1 ratio by weight, was casted or spin coated and cured at $60^{\circ} \mathrm{C}$. Peeling-off of very thin PDMS films $(\sim 100 \mu \mathrm{m})$ required the deposition of a thin Cr layer $(2 \mathrm{~nm})$ followed by Au layer $(6 \mathrm{~nm})$ using an ebeam evaporator prior to PDMS application to reduce the adhesion between the $\mathrm{SiO}_{2}$ and the PDMS. To guarantee the final cleanliness of the CNT film, half cured PDMS films $\left(60{ }^{\circ} \mathrm{C}\right.$ for $5 \mathrm{~min}$ ) were used in a state of a viscous polymer and were applied as an adhesive film onto the CNTs. The use of partially cured films substantially reduced wetting of the CNTs. Finally, a passivation PDMS membrane with predefined holes and the CNT flexible circuit were bonded. The PDMS passivation layer was prepared using a SU83050 (MICRO-CHEM) patterned mold (see Supplementary Fig. 1). PDMS passivation was bonded using a custom made holder mounted on a microscope stage. PDMS-PDMS bonding was promoted by oxygen plasma treatment to both films. Oxidation of PDMS surface exposes silanol groups ( $\mathrm{Si}-\mathrm{OH})$ so when the two films are brought together they form covalent siloxane bonds ( $\mathrm{Si}-\mathrm{O}-\mathrm{Si}$ ) which provide excellent sealing (Duffy et al. 1998). Bonding with polyimide and parylene $C$ substrates was achieved by means of an intermediate thin layer of liquid PDMS followed by curing at $60{ }^{\circ} \mathrm{C}$. Finally, the medical tape was bonded with the passivation by exploiting the adhesiveness of the tape. These processes yielded $30-65 \%$ clean and capacitive electrodes utilizing an entirely manual preparation. We expect that mechanizing the process can dramatically improve the yield. For electrophysiological experiments the flexible CNT MEA was mounted on a PCB $\left(49 \times 49 \mathrm{~mm}^{2}\right)$ with 60 $\mathrm{Au}$ traces and contact pads. A glass chamber was mounted on top of the PCB using uncured PDMS. 


\subsection{Electrical resistance measurements of CNT films}

Sets of CNT bars with different lengths and constant width and height, were fabricated between TiN pads as follows. A $100 \mathrm{~nm}$ TiN layer was sputtered (MRC RF sputter) on a $\mathrm{Si} / \mathrm{SiO}_{2}$ support followed by lithography and reactive ion etching (Nextral 860) to pattern the TiN pads. Due to a marked difference between the diffusion rate of $\mathrm{Ni}$ through $\mathrm{SiO}_{2}$ and $\mathrm{TiN}$ at the CNT growth temperature, two layers of $\mathrm{Ni}$ were deposited by an e-beam evaporator (VST). The first layer $(8 \mathrm{~nm})$ was deposited on the inner half of the TiN pads and the second layer $(2.5 \mathrm{~nm})$ between the TiN pads on the $\mathrm{SiO}_{2}$ substrate. Finally a CNT film was grown by CVD (for detailed illustration see Supplementary Fig. 2). Current versus voltage screen of the different length TiN-CNTs-TiN bars was recorded and their electrical resistance was calculated (for details see Supplementary Fig. 3).

\subsection{Electrochemical analysis}

The electrochemical properties of the CNT electrodes were characterized by performing cyclic voltammetry (CV) and electrochemical impedance spectroscopy (EIS) in PBS. An $\mathrm{Ag} / \mathrm{AgCl}$ electrode served as a reference electrode and a platinum wire as a counter electrode. CV measurements were conducted using a potentiostat (263A Princeton Applied Research) under ambient conditions and recorded using the PowerCV software (Princeton Applied Research). The DC capacitance was derived from the oxidation current versus the scan rate data according to the relation: $\mathrm{i}=\mathrm{C} \cdot d V / d t$ in which $\mathrm{i}$ is the charging current, $\mathrm{C}$ is the DC capacitance and $d V / d t$ is the scan rate. EIS measurements were conducted under equilibrium conditions by applying small $(10 \mathrm{mV}) \mathrm{AC}$ signals over the frequency range of $1 \mathrm{~Hz}$ to $10 \mathrm{kHz}$ using a lock-in amplifier (SR830, Stanford Research Systems) and a potentiostst (263A, Princeton Applied Research).

\subsection{Retina preparation and handling}

Embryonic chick retinas (day 14) were isolated and transferred to the experimental chamber, placed RGC layer down onto the flexible MEAs. Better coupling between the tissue and the electrodes was achieved by placing a small piece of polyester membrane filter $(5 \mu \mathrm{m}$ pores; Sterlitech, Kent, WA, USA) on the retina followed by a ring weight which served as a slice anchor holder. Retinas were kept at physiological conditions according to a previously reported protocol (Hammerle et al. 1994) with temperature of $34{ }^{\circ} \mathrm{C}$ and perfuse $(2-5 \mathrm{ml} / \mathrm{min}$ ) with oxygenated artificial cerebro-spinal fluid.

\subsection{Electrical recording}

Neuronal electrical signals were amplified (gain $\times 1,200$, MultiChannel Systems MEA1060-Inv, Reutlingen, Germany), digitized using a 128-channel analogue to digital converter
(MultiChannel Systems MC_Card, Reutlingen, Germany) and recorded (MultiChannel Systems MC_Rack, Reutlingen, Germany). All additional signal analysis was performed using Matlab software (MathWorks). Electrically stimulated neuronal activity was digitized at $20 \mathrm{kHz}$ and spikes were detected by setting a threshold of signal to noise ratio (SNR) SNR $>4$ (related to the pre-stimulation noise level). Due to amplifier saturation artifact, the period of $20 \mathrm{~ms}$ post stimulation was ignored. The response of the retinal site to electrical stimulation was defined as the detected spikes count.

\subsection{Electrical stimulation}

Chick retinas were electrically stimulated using a dedicated stimulator (STG-1008, Multi-Channel Systems, Reutlingen, Germany) through one of the MEA electrodes each time (versus an external reference) with charge-balanced biphasic (cathodic first) current stimulation (pulse width: $1 \mathrm{~ms}$ and pulse amplitude: 1-10 $\mu \mathrm{A}$ ). Each stimulation session included stimulations at the entire intensity range (increased by $1 \mu \mathrm{A}$ every $10 \mathrm{~s}$ ) and was repeated five times. To validate that the electrical stimulation resulted from synaptic processes, synaptic blockers CNQX (Sigma) and APV (Sigma) were applied (75 $\mu \mathrm{M}$ and $400 \mu \mathrm{M}$ respectively).

\section{Results}

\subsection{All-CNT flexible MEA fabrication}

We investigated a new fabrication technique utilizing a combination of micro and nano schemes to realize non-Faradaic CNT based electrodes with very high specific capacitance using a simple fabrication process. To support a simple and robust fabrication process, the electrodes are made exclusively of CNTs so no complex fabrication integration was required. The general fabrication process, described in Fig. 1a, is based on loosely-bound MWCNT films grown using CVD process from a thin Ni layer (Fig. 1a-2). The Ni layer is deposited on a support $\mathrm{Si} / \mathrm{SiO}_{2}$ substrate (Fig. 1a-1). An uncured polymer (e.g. PDMS or polyimide) is then casted on the substrate with the CNT film. After curing, the CNTs are integrated with the polymer. The polymer and the CNT films can then be peeled-off from the surface (Fig. 1a-3). Similar results can be obtained by applying an adhesive tape against the CNT pattern or by using vapor deposition of Parylene C. The CNT carrying film and a second layer of holey PDMS membrane are then bonded together (Fig. 1a-4) to form a flexible circuit containing passivated CNT conducting tracks and exposed CNT electrodes. The biocompatibility of PDMS, parylene $\mathrm{C}$ and polyimide is well established. Polyimide and parylene $C$ have comparable elastic moduli of $\sim 2-4 \mathrm{GPa}$ (two to three orders of magnitude lower than that of metal and 
Fig. 1 All-CNT flexible multielectrode arrays. a Electrode fabrication scheme. (1) The process is based on a single photolithographically defined $\mathrm{Ni}$ catalyst layer. (2) The CNT film is then grown using a CVD process. (3) Next, the film is transferred to a polymeric support (e.g. medical adhesive tape, PDMS, Parylene C, polyimide). (4) Finally, a second polymeric layer (PDMS) with predefined holes is bonded with the CNT carrying film for passivation. b Different patterns of flexible CNT electrode arrays on different support layers: (1) PDMS, (2) medical adhesive tape, (3) Parylene C and (4) polyimide (a)

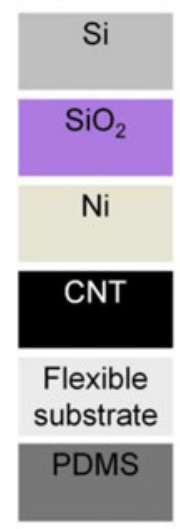

(b)

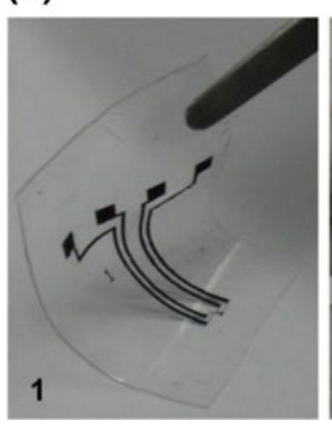

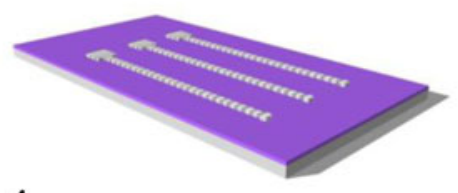

1

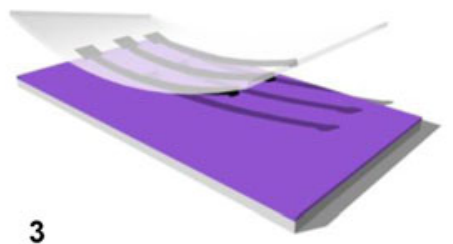

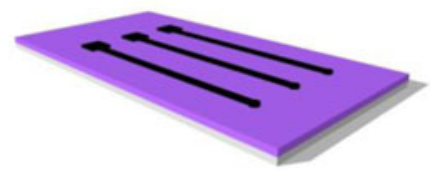

2

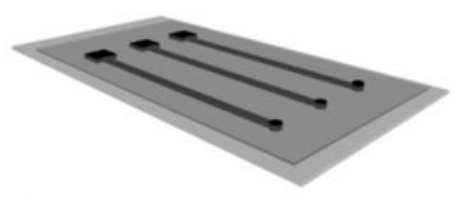

4
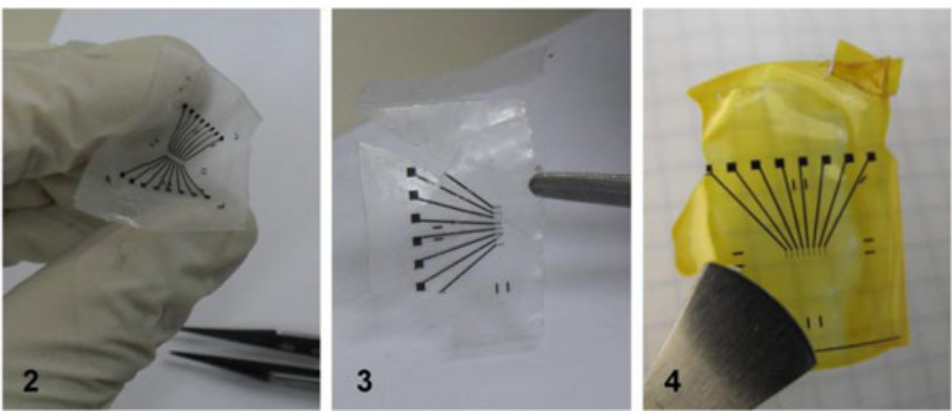

silicon), while PDMS elasticity (depending on preparation conditions) can be further reduced down to $\sim 0.05 \mathrm{MPa}$ (Rousche et al. 2001; Brown et al. 2005; Rodger et al. 2008; Meacham et al. 2011). Polyimide can be patterned using standard microfabrication such as photolithography and reactive ion etching (Cheung et al. 2007; Mercanzini et al. 2008) and parylene $\mathrm{C}$ has superior resistance to moisture. Finally, the adhesive medical tape enables quick and simple fabrication with well exposed CNT films. Such films may be well suited for skin-applied electrode arrays.

The process is general enough to include additional layers for multi-layer stacking, as well as to incorporate additional elements such as photodiodes. Photodiodes integration with CNT electrode array would enable neuronal stimulation using light, a desirable feature in retinal implants aimed at substituting degenerated photoreceptors.

This scheme has several notable advantages over previously proposed concepts. Foremost, it is simple for implementation, requiring only two independent lithographic steps. Unlike dispersion methods, the use of standard lithography allows high resolution patterning of the CNT film and a simple integrating of the CNT pattern with the polymer substrate. Moreover, the entire device it based only on very few elementary fabrication steps. Additionally, the device benefits from strong overall stability against peeling and degradation due to seamless integration between the electrodes and conducting traces. Finally and most importantly, at no stage of the process, the surfaces of the CNT electrodes are exposed to any solvents, photo-resists, or electro-plating baths rendering the entire process very clean, and therefore ensuring the non-Faradaic nature of the electrodes.

While the process described above appears to be straight forward, two critical properties must be carefully maintained to guarantee proper function of the end device. Foremost, is the high effective surface area of the electrodes. Clean CNTs have outstanding electrochemical properties, however, impurities and polymeric residues can dramatically hamper the proper operation of the electrodes. Indeed, we have noticed that the cleanliness of the electrode surface can be compromised if the polymer (e.g. PDMS) penetrates the CNT film. The second critical requirement is the electrical conductivity of the CNT interconnects.

\subsection{Characterization of flexible CNT devices}

We begin by discussing the cleanliness of the CNT films and their electrochemical properties. We found that different polymers and deposition methods (e.g. spin coating, applying adhesive tape, and vapor deposition) dramatically affect the extent of the polymer penetration into the film. Accordingly, careful validation of the morphological and electrochemical properties of the electrodes is important. Validation was achieved by using electrode arrays with different electrode diameter $(100,150$, 
$200,250,300,350,400$ and $450 \mu \mathrm{m})$. Electrode arrays were realized following the scheme depicted in Fig. 1a and were then systematically tested. The CNT film cleanliness was first validated qualitatively using environmental scanning electron microscopy (ESEM). Figure 2a shows ESEM images of a typical CNT surface on a medical adhesive tape (Fig. 2a-1), on Parylene C (Fig. 2a-2) and on PDMS (Fig. 2a-3). While part of the CNT film is embedded in the cured PDMS, the top surface of the CNTs is clearly exposed (Fig. 2a-3). Apparently clean, highly intertwined MWCNTs were observed on the medical tape and on the Parylene $\mathrm{C}$ surfaces (Fig. 2a-1 and a-2). Cross section image of the CNT film on a medical adhesive tape (Fig. 2a-1, inset) demonstrates a CNT film on top of the flexible medical tape substrate. Under proper preparation conditions clean CNT films were reliably transferred to all different flexible substrates described above (see Section 2).

The ESEM imaging was followed by electrochemical characterization using CV that records current resulting from
Fig. 2 Electrochemical and transport properties of CNT devices. a An ESEM image of MWCNTs on a medical adhesive tape; Inset: a zoom out ESEM cross section image of a MWCNT film on a medical adhesive tape (marked with arrow) (1), Parylene C (2), and PDMS (3), scale bar: $2 \mu \mathrm{m}$; Inset scale bar: $100 \mu \mathrm{m}$. b $\mathrm{CV}$ scans of a CNT electrode $(100 \mu \mathrm{m}$ in diameter) at different scan rates with blue, red and black lines corresponding to scan rates of 15,50 and $150 \mathrm{mV} / \mathrm{s}$ respectively. c Charging current versus scan rate of a CNT electrode (100 $\mu \mathrm{m}$ in diameter), solid line is a linear fit. d CNT electrode capacitance versus electrode surface area, solid line is a linear fit. Inset: Microscope image of CNT electrodes (100, 150,200 and $250 \mu \mathrm{m}$ in diameter). Measurements shown are for a single representative set of devices. e CNT electrode (100 $\mu \mathrm{m}$ in diameter) impedance versus frequency. All electrochemical measurements were performed in PBS with an $\mathrm{Ag} / \mathrm{AgCl}$ reference electrode. $\mathbf{f}$ Raman spectrum of a MWCNT film. g CNT film electrical resistance versus number of squares. Inset: Microscope image of different length TiN-CNTs-TiN bars used to derive film electrical resistance. Measurements shown are for a single representative set of devices (a)
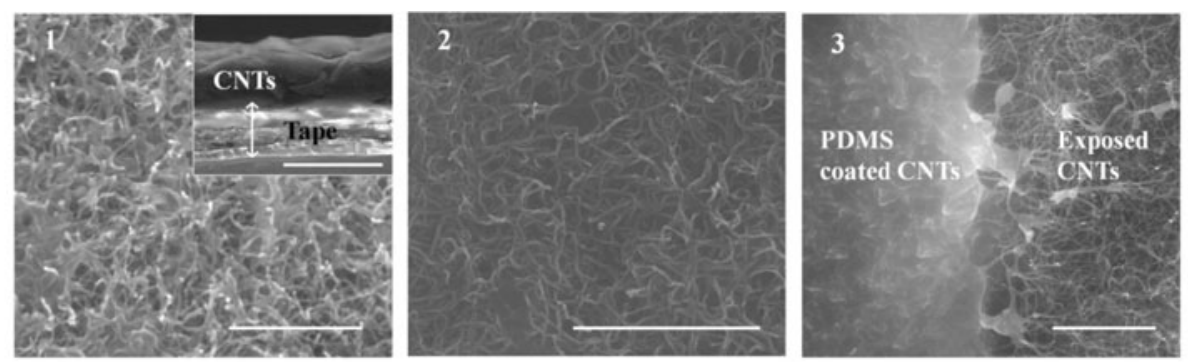

(b)

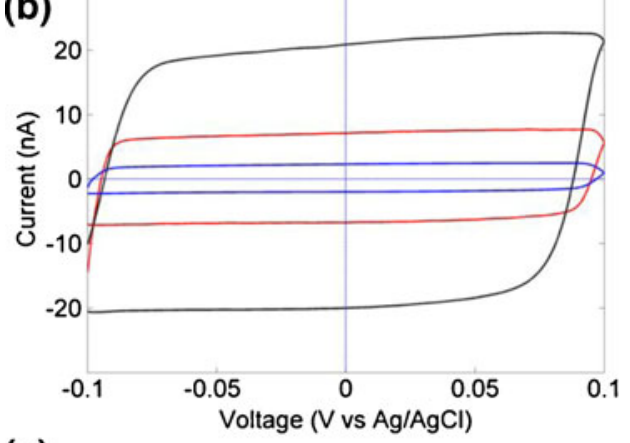

(c)

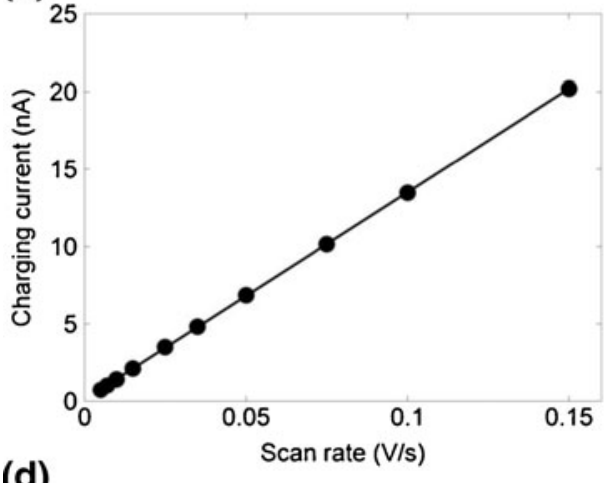

(d)

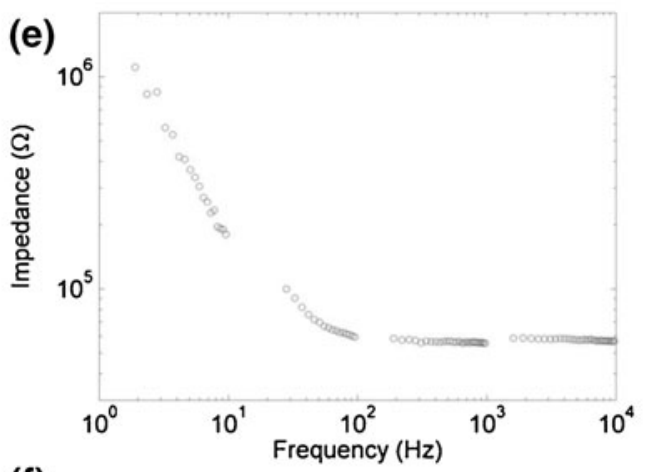

(f)

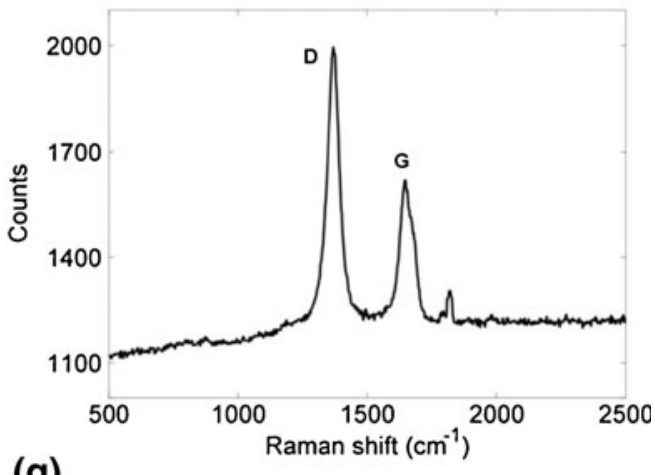

(g)
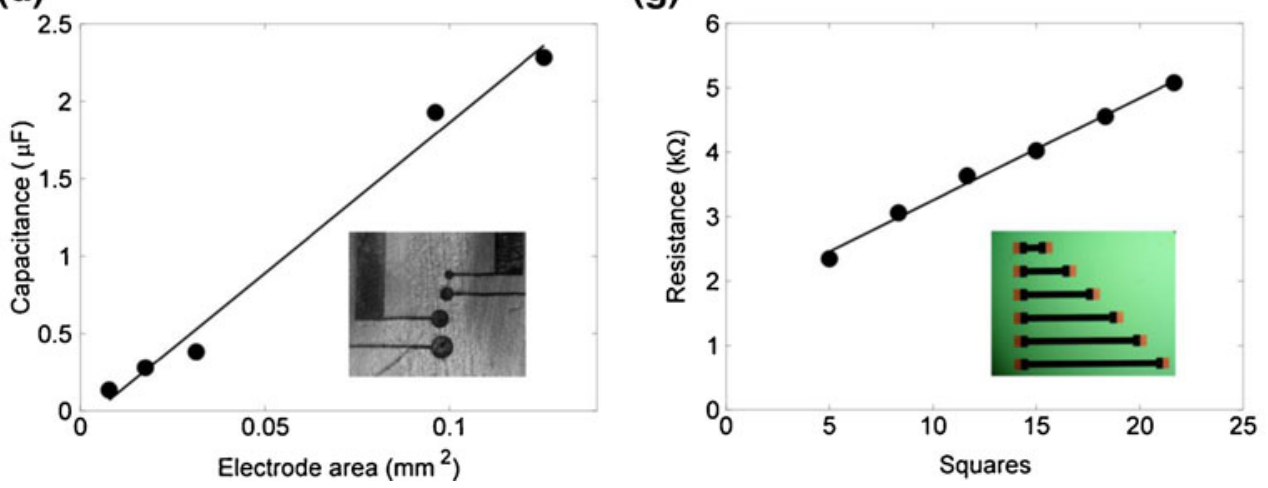
scanning the applied voltage, and EIS, which measures frequency-dependent changes in the impedance. CV and EIS measurements were performed with a three-electrode cell configuration using phosphate buffered saline (PBS) and $\mathrm{Ag} / \mathrm{AgCl}$ reference electrode. The CV data (Fig. 2b) is markedly flat, showing no signs of reactivity, as expected from clean CNT electrodes (Gabay et al. 2007). Current versus scan-rate plots show clear linear dependence (Fig. 2c) in accordance with a double layer capacitor model. Finally, the capacitance of different size electrodes was calculated and plotted and the specific capacitance value was derived, yielding values as high as 2 $\mathrm{mFcm}^{-2}$ (Fig. 2d). Variation of the impedance with frequency $(1 \mathrm{~Hz}$ to $10 \mathrm{kHz})$ is presented in Fig. 2e. The impedance of a $100 \mu \mathrm{m}$ diameter CNT electrode (including its long conducting trace) at biologically relevant frequency for neural recording of $1 \mathrm{kHz}$ is $55 \mathrm{k} \Omega$. The electrochemical measurements were also used as a tool to directly quantify the extent of the clean surface. Sensitive surface analytical methods such as X-ray photoelectron spectroscopy (XPS) could also be used as complementary tool to electrochemical measurements.

Since the CNT films also constitute the circuit lines of our devices, their electrical resistance is consequential (Agrawal et al. 2007). The electrical performances of MWCNTs depend on many factors such as average length, diameter, wall number, structural defects, film thickness, and the amount of amorphous carbon (Ferrari and Robertson 2000). While some of these parameters can be controlled in the growth process to optimize the conductivity of the films, CNT films generally suffer from poor conductivity compared with typical metals. We note that for our device needs, owing to the large electrode-solution impedance, exceptionally high trace conductivity is not critically important and values in the order of several kilo ohms are acceptable.

To validate the CNT film quality and to quantify the electrical conductivity values, CNT films were characterized using Raman spectroscopy (RS) and direct electrical measurements respectively. RS was performed to characterize the nature and the quality of the MWCNT films (Fig. 2f). Raman spectrum of the CNT films show two distinct peaks at 1,360 (D-band) and 1,580 cm-1 (G-band) (Thomsen and Reich 2007). We used the ratio between the $D$ and the $G$ band $\left(\mathrm{I}_{\mathrm{D}} / \mathrm{I}_{\mathrm{G}}\right)$ as a crude characterization of the defect density and each CNT film was measured at 20 different sites. The $\mathrm{I}_{\mathrm{D}} / \mathrm{I}_{\mathrm{G}}$ for all films was higher than one, indicating fairly poor film quality associated with the highly entangled CNTs. However, we have extensively used similar films in the past to perform recording from dissociated neurons (Gabay et al. 2007; Shein et al. 2009) and from mouse retina (Shoval et al. 2009) with excellent results. The obtained films are thus very well suited for neuronal stimulation. To validate the durability of the CNT films upon mechanical stress we have tested the electrical properties of the CNT films following repeated cycles (up to 30 cycles) of folding and winding. No significant change in film resistivity was identified, during or after these manipulations. A major concern when considering the biocompatibility of the CNT electrodes is Ni traces and we have tested our CNT electrodes for $\mathrm{Ni}$ traces and performed biocompatibility tests by culturing rat cortical cells on the CNT films (according to a previously reported protocol (Shein et al. 2009)). We have conducted energy-dispersive $\mathrm{x}$-ray spectroscopy (EDS) tests that revealed very small residues of Ni. Apparently $\mathrm{Ni}$ is effectively embedded in the CNTs and has no adverse effects.

Finally, to reliably measure the electrical resistance of the MWCNT traces, a special testing scheme was implemented. Sets of different length MWCNT bars (width and height remained constant) were fabricated (Supplementary Fig. 2) with TiN contacts (TiN-CNTs-TiN). The TiN pads are instrumental to achieve reliable Ohmic contacts to the CNT films, guarantying consistent measurements. It should be noted that while the contact resistance of TiN is substantial, TiN is a conducting material most suitable for CNT growth under the high temperature of the CVD process and therefore is a very convenient material to perform the film resistance validation discussed here. Current versus voltage trace for each TiN-CNTs-TiN bar was recorded and the electrical resistance was calculated. All samples exhibited an Ohmic behavior with values ranging between 2 and $15 \mathrm{k} \Omega$. To derive their sheet resistance, electrical resistance values were plotted versus the number of squares in each bar (Fig. 2g; for explanation on sheet resistance calculations see Supplementary Fig. 3). Values ranging between 160 and 1,850 $\Omega / \square$ for different CVD growth conditions of the MWCNT film were obtained. Owing to the high electrode-electrolyte impedance values, we conclude that the CVD grown MWCNT films are conducting well enough to be readily used as effective conducting traces for our application.

\subsection{Extracellular neuronal recording and stimulation} using the flexible CNT MEA

Having established the electrical as well as the electrochemical properties of the CNT films, we now turn to describe the electrophysiological performances of the flexible electrodes. An electrode array compatible with a standard multi-electrode array recording and stimulation setup was realized on a printed circuit board (PCB) support (Fig. 3a). The array consists of 16 electrodes on a medical tape support each connected to an external pad. A top PDMS passivation layer, $150 \mu \mathrm{m}$ thick and with $50 \mu \mathrm{m}$ diameter holes, was used to define the effective size of the electrodes (Fig. 3a, inset). The flexible array was then mounted onto the PCB carrier to accommodate the link between the electrodes and external amplifiers. A glass cylinder was glued to the PCB support to serve as a well for the physiological medium.

Embryonic chick retina (day 14) was used as a neuronal model. The retina was extracted and transferred to the medium chamber under physiological conditions. The retina was then 
Fig. 3 Flexible CNT MEA for extracellular neuronal recording and stimulation. a The flexible CNT electrode array mounted on a PCB support linking the electrodes to external amplifiers (scale bar: $5 \mathrm{~mm}$ ). The array consists of 16 electrodes and a top passivation layer with $50 \mu \mathrm{m}$-diameter holes which define the electrode effective size. An embryonic chick retina (day 14) was flattened on the electrode array. The edge of the retina is marked with a dashed line. Inset: enlargement of the electrodes area (area marked with a solid line; scale bar: $200 \mu \mathrm{m}$ ). b A circuit model for extracellular recording and stimulation from a neural tissue using the flexible electrode array. The model demonstrates the electrochemical interface resistance and capacitance of the CNT electrode and the solution derived shunt capacitance as well as the point of stimulation (a)

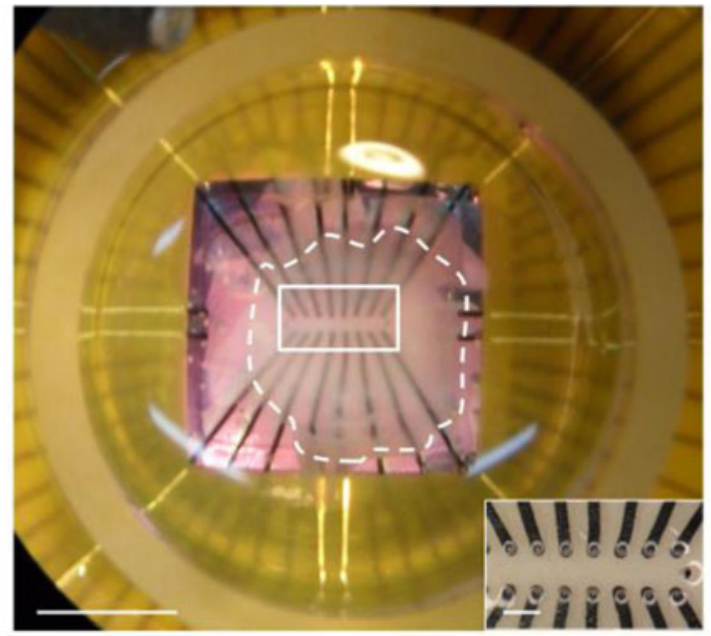

(b)

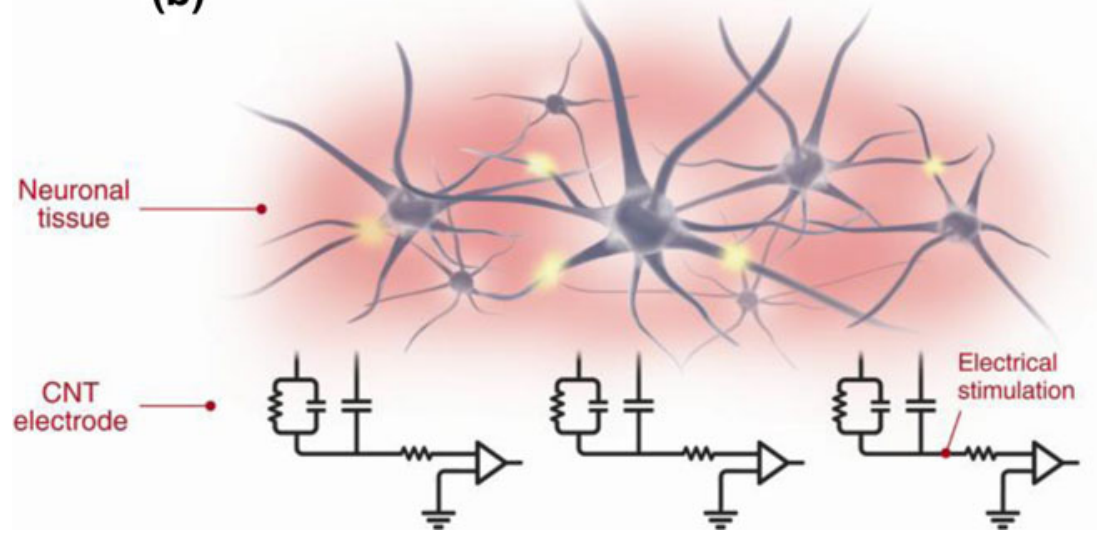

flattened on the electrode array (Fig. 3a), with the retinal ganglion cell (RGC) layer facing down (as in an epi-retinal implant) and was anchored with a weight. Figure 3 b illustrates a circuit model for extracellular recording and stimulation of neuronal tissue using the micro electrode array, depicting the electrochemical interface resistance and the capacitance of the CNT electrode as well as the solution derived shunt capacitance and the stimulation point.

At day 14 the embryonic retina is still at an early developmental stage and clear spontaneous activity waves were recorded demonstrating the overall functionality of the device and the setup. We next tested the CNT electrodes suitability to evoke electrical activity in the retina tissue. Stimulation was achieved at currents as low as $4 \mu \mathrm{A}$ (Fig. $4 \mathrm{a}$ and b) and stimulation pulse width of $1 \mathrm{~ms}$. With nearly perfectly capacitive electrodes, these values are well within the limits of safe stimulation. The observed electrical response is typical for pre-synaptic cells activation. Validation of the synaptic processes was achieved with the use of the synaptic blockers 6cyano-7-nitroquinoxaline-2,3-dione (CNQX) and 2-amino-5phosphonovaleric acid (APV). $400 \mathrm{~s}$ after the introduction of the synaptic blockers no retinal ganglion cell activation was measured (Fig. 4c).

\section{Discussion and conclusions}

We can now turn to look at how our new CNT electrodes rank compared with previously reported technologies. Table 1 summarizes specific DC capacitance, stimulation threshold and SNR values obtained with other CNT and flexible electrode technologies. The table refers to studies that demonstrated either recording or stimulation of neuronal activity. DC capacitance values of $1-10 \mathrm{mFcm}^{-2}$ were measured from most CNT electrodes on both rigid and flexible substrates. The all CNT flexible MEA presented in this study is well within this range with $2 \mathrm{mFcm}^{-2}$, exceeding both CNT electrodes grown directly on flexible polyimide with $0.1 \mathrm{mFcm}^{-2}$ (Hsu et al. 2010; Chen et al. 2011b) as well as Pt electrodes coated with SWCNT (drop coating) on a rigid Pyrex substrate with $4.5 \cdot 10^{-6} \mathrm{mFcm}^{-2}$ (Gabriel et al. 2009). It should be noted that SNR and stimulation threshold values depend on the examined tissue as well as on the size and shape of the electrode. Therefore they cannot be used as a direct measure of MEA devices. The SNR in particular, provides only a validation for the acceptable performance of the electrodes. A stimulation threshold of $4 \mathrm{nC}$ measured by our flexible CNT MEA is lower than that reported by other CNT MEA 
(a)

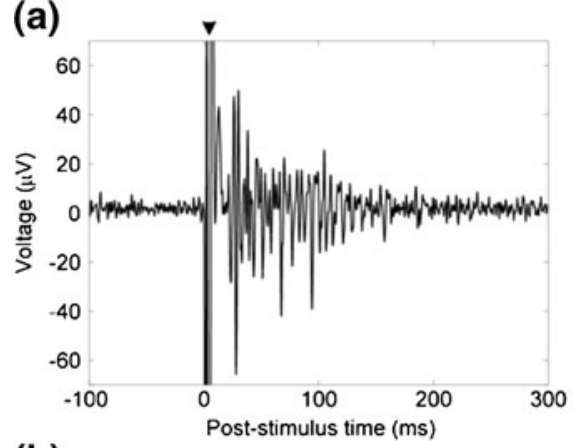

(b)
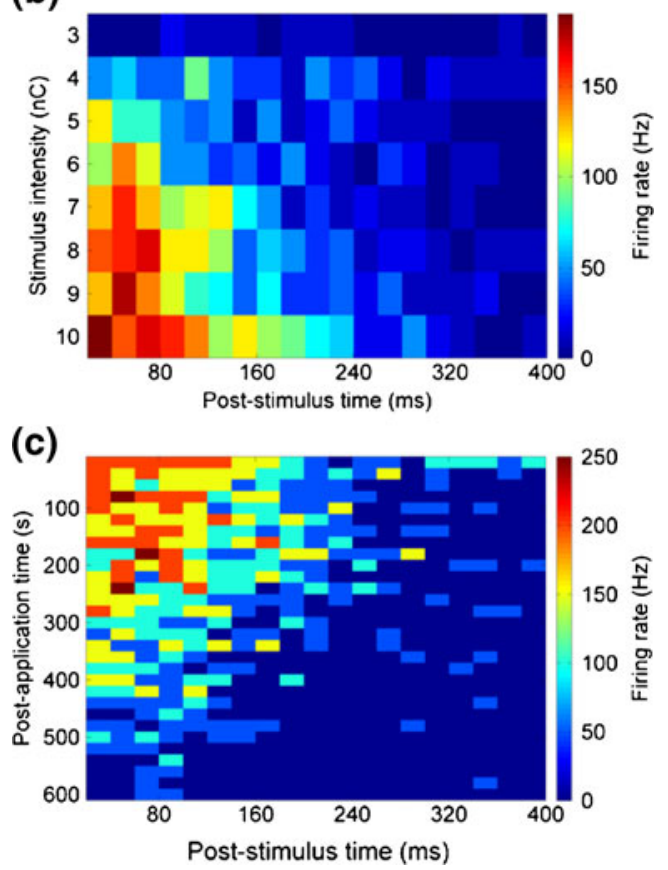

Fig. 4 Electrical recording and stimulation of chick retina with flexible CNT MEA. a Evoked activity using a biphasic cathodic first pulse (arrowhead). The large signal at $\mathrm{t}=0$ is an artifact of the stimulation. b Firing rate of evoked activity at different stimulation intensities (3-10 $\mathrm{nC})$. c Firing rate of evoked activity after synaptic blockers CNQX and APV application (stimulation was applied every $10 \mathrm{~s}$ ). After $400 \mathrm{~s}$ no retinal ganglion cells activation is observed

technologies (both rigid and flexible) and is similar to values obtained with TiN commercial devices, further demonstrating the overall high quality of our devices.

We have shown that our new flexible all CNT MEA performances are equivalent to rigid $\mathrm{CNT}$ technologies with the obvious major advantage of being flexible. Two flexible CNT technologies used for neuronal recording and stimulation were reported before. Lin and co-workers fabricated a vertically aligned CNT (VACNT) MEA embedded in Parylene-C film (Lin et al. 2009) while Hsu and co-workers used low temperature CVD (i.e. $400{ }^{\circ} \mathrm{C}$ ) to directly grow CNT MEA on polyimide (Hsu et al. 2010; Chen et al. 2011b). The specific capacitance of our CNT MEA is significantly higher than that of the directly grown CNTs on polyimide. Compared with both rigid and flexible technologies, our device benefits from the advantages of a very clean and simple fabrication scheme and most importantly a seamless integration between the electrode and the circuit, ultimately supporting a reliable and scalable fabrication of state of the art flexible MEAs. To conclude this discussion, our novel flexible CNT electrodes, as other clean carbon based electrodes, are distinguished by having a clear capacitive nature. Being produced by a simple, clean and robust process, these electrodes properties surpass previously described technologies.

Notwithstanding these promising results, some improvements in the fabrication scheme are desirable. For example, the $150 \mu \mathrm{m}$ insulation layer locates our electrodes at a significant distance from the tissue, limiting the spatial resolution of the device as reflected in the relative low amplitude of the recorded signals (Fig. 4a). Reducing the thickness of the insulation layer will also improve the adhesion of the tissue to the electrodes, further promoting the spatial resolution.

To summarize, a new scheme based on CNTs was presented and demonstrated as an advantageous approach to form high performance neuronal electrode array devices. The electrodes gain their performances from the combination of several different CNT properties. Foremost, CNTs films have extremely large surface area making them very effective electrochemical electrode with capacitive charge injection mechanism. CNTs are also inert and strong, making the electrodes stable in biological conditions. As CNT films are suitable to withhold bending, they are very well suited for flexible electronic applications. In the realm of multi-electrode arrays, this feature is particularly important as flexible MEA devices are of great interest for implantable applications. Unlike other coatings that may tend to crack and disconnect from the flexible substrate during bending, CNT films are durable owing to their remarkable mechanical properties and the unique structure of the MWCNTs film. The entangled bundles of tubes, forming a dense and continuous yet porous film, make these films particularly optimal for neuronal applications. Additionally, the adhesion between the CNTs and the polymeric substrate is strong, making the CNT film an integrated part of the substrate. Since CNTs are chemically inert they are also durable against corrosion, a very common challenge in conventional metal technology in biological applications. The circuit structure is seamless and all elements, connecting pads, conducting traces and electrodes are made of CNT. This is an enormous advantage for both in vivo and in vitro long term use since it eliminates delamination of the coatings and the formation of cracks. These cracks result with leakage currents and failure of the device as often occurs with layering and connection of different materials. All these properties are added to the relatively simple and robust fabrication process discussed above. This fabrication process can be easily extended to include elements such as photodiodes and allows for stacking of different functionality layers, make the all-carbon- 


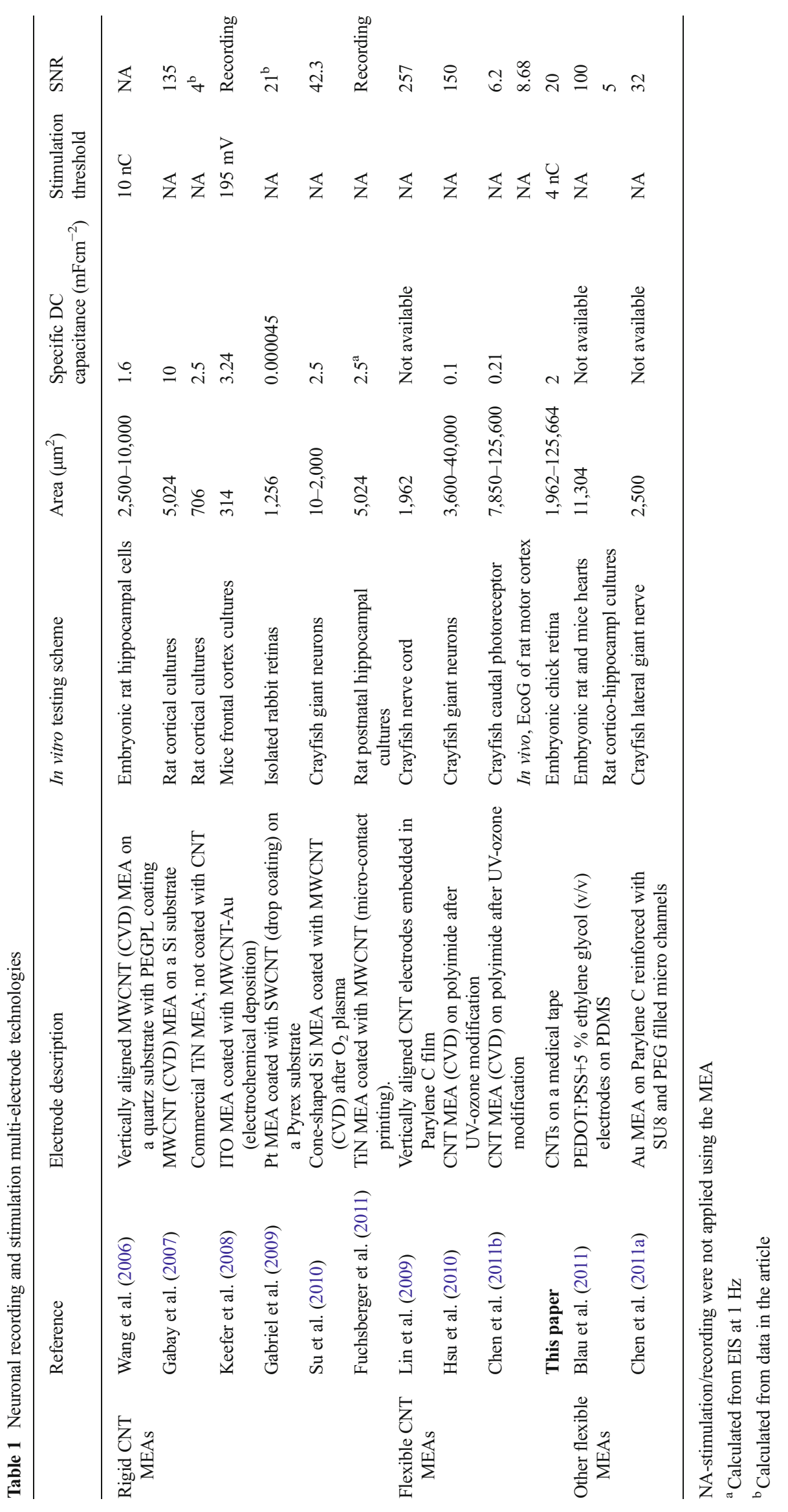


nanotube flexible neural electrodes, presented here, a promising element in future neuro-prosthetic devices.

Acknowledgments The authors thank Nurit Atar for providing the polyimide substrates. Micro and nano fabrication and characterization were performed at Tel Aviv University Center for Nanoscience and Nanotechnology. We also acknowledge the support of a grant from the Israel Ministry of Science and Technology, the Israel Science Foundation and the European Research Council funding under the European Community's Seventh Framework Program (FP7/2007- 2013)/ERC grant agreement FUNMANIA-306707.

Open Access This article is distributed under the terms of the Creative Commons Attribution License which permits any use, distribution, and reproduction in any medium, provided the original author(s) and the source are credited.

\section{References}

M.R. Abidian, J.M. Corey et al., Conducting-polymer nanotubes improve electrical properties, mechanical adhesion, neural attachment, and neurite outgrowth of neural electrodes. Small 6(3), 421$429(2010)$

C. Adams, K. Mathieson et al., Development of flexible arrays for in vivo neuronal recording and stimulation. Nucl. Inst. Methods Phys. Res. A 546(1-2), 154-159 (2005)

S. Agrawal, M. S. Raghuveer et al., Defect-induced electrical conductivity increase in individual multiwalled carbon nanotubes. Appl. Phys. Lett. 90(19) (2007)

A. Blau, A. Murr et al., Flexible, all-polymer microelectrode arrays for the capture of cardiac and neuronal signals. Biomaterials 32(7), 1778-1786 (2011)

X.Q. Brown, K. Ookawa et al., Evaluation of polydimethylsiloxane scaffolds with physiologically-relevant elastic moduli: interplay of substrate mechanics and surface chemistry effects on vascular smooth muscle cell response. Biomaterials 26(16), 3123-3129 (2005)

D. Carnahan, D. Esposito et al., Direct growth of individual carbon nanotubes on a flexible plastic substrate via selective heating. Nanotechnology 21(22) (2010)

S.K. Chang-Jian, J.R. Ho et al., A flexible carbon nanotube field emitter fabricated on a polymer substrate by a laser separation method. Solid State Commun. 150(13-14), 666-668 (2010)

Y.Y. Chen, H.Y. Lai et al., Design and fabrication of a polyimide-based microelectrode array: application in neural recording and repeatable electrolytic lesion in rat brain. J. Neurosci. Methods 182(1), 6-16 (2009)

C.H. Chen, S.C. Chuang et al., A three-dimensional flexible microprobe array for neural recording assembled through electrostatic actuation. Lab Chip 11(9), 1647-1655 (2011a)

Y. C. Chen, H. L. Hsu et al., An active, flexible carbon nanotube microelectrode array for recording electrocorticograms. J. Neural Eng. 8(3) (2011b)

K.C. Cheung, P. Renaud et al., Flexible polyimide microelectrode array for in vivo recordings and current source density analysis. Biosens. Bioelectron. 22(8), 1783-1790 (2007)

S.F. Cogan, Neural stimulation and recording electrodes. Annu. Rev. Biomed. Eng. 10, 275-309 (2008)

D.C. Duffy, J.C. McDonald et al., Rapid prototyping of microfluidic systems in poly(dimethylsiloxane). Anal. Chem. 70(23), 4974 4984 (1998)
A.C. Ferrari, J. Robertson, Interpretation of Raman spectra of disordered and amorphous carbon. Phys. Rev. B 61(20), 14095-14107 (2000)

A.A. Fomani, R.R. Mansour, Fabrication and characterization of the flexible neural microprobes with improved structural design. Sensors Actuators B Phys. 168(2), 233-241 (2011)

K. Fuchsberger, A. Le Goff et al., Multiwalled carbon-nanotubefunctionalized microelectrode arrays fabricated by microcontact printing: platform for studying chemical and electrical neuronal signaling. Small 7(4), 524-530 (2011)

T. Gabay, M. Ben-David et al., Electro-chemical and biological properties of carbon nanotube based multi-electrode arrays. Nanotechnology 18(3) (2007)

G. Gabriel, R. Gomez et al., Easily made single-walled carbon nanotube surface microelectrodes for neuronal applications. Biosens. Bioelectron. 24(7), 1942-1948 (2009)

O. Graudejus, Z. Yu et al., Characterization of an elastically stretchable microelectrode array and its application to neural field potential recordings. J. Electrochem. Soc. 156(6), 85-94 (2009)

O. Graudejus, B. Morrison et al., Encapsulating elastically stretchable neural interfaces: yield, resolution, and recording/stimulation of neural activity. Adv. Funct. Mater. 22(3), 640-651 (2012)

H. Hammerle, U. Egert et al., Extracellular recording in neuronal networks with substate integrated microelectrode arrays. Biosens. Bioelectron. 9(9-10), 691-696 (1994)

H.L. Hsu, I.J. Teng et al., Flexible UV-ozone-modified carbon nanotube electrodes for neuronal recording. Adv. Mater. 22(19), 2177-2181 (2010)

E.W. Keefer, B.R. Botterman et al., Carbon nanotube coating improves neuronal recordings. Nat. Nanotechnol. 3(7), 434-439 (2008)

J.D. Klein, S.L. Clauson et al., Morphology and charge capacity of sputtered iridium oxide films. J. Vac. Sci. Technol., A 7(5), 3043-3047 (1989)

S.P. Lacour, S. Benmerah et al., Flexible and stretchable micro-electrodes for in vitro and in vivo neural interfaces. Med. Biol. Eng. Comput. 48(10), 945-954 (2010)

C.M. Lin, Y.T. Lee et al., Flexible carbon nanotubes electrode for neural recording. Biosens. Bioelectron. 24(9), 2791-2797 (2009)

G.E. Loeb, R.A. Peck et al., Toward the ultimate metal microelectrode. J. Neurosci. Methods 63(1-2), 175-183 (1995)

K.A. Ludwig, J.D. Uram et al., Chronic neural recordings using silicon microelectrode arrays electrochemically deposited with a poly $(3,4-$ ethylenedioxythiophene) (PEDOT) film. J. Neural Eng. 3(1), 59-70 (2006)

K.W. Meacham, L. Guo, S.P. De Weerth, P. Hochman, Selective stimulation of the spinal cord surface using a stretchable microelectrode array. Front. Neuroeng. 4, 12 (2011)

A. Mercanzini, K. Cheung et al., Demonstration of cortical recording using novel flexible polymer neural probes. Sensors Actuators B Phys. 143(1), 90-96 (2008)

D.R. Merrill, M. Bikson et al., Electrical stimulation of excitable tissue: design of efficacious and safe protocols. J. Neurosci. Methods 141(2), 171-198 (2005)

S. Myllymaa, K. Myllymaa et al., Fabrication and testing of polyimidebased microelectrode arrays for cortical mapping of evoked potentials. Biosens. Bioelectron. 24(10), 3067-3072 (2009)

L.S. Robblee, J.L. Lefko et al., Activated IR - an electrode suitable for reversible charge injection in saline solution. J. Electrochem. Soc. 130(3), 731-733 (1983)

D. Robinson, The electrical properties of metal electrodes. Proc. IEEE 56(6), 7 (1968)

D.C. Rodger, A.J. Fong et al., Flexible parylene-based multielectrode array technology for high-density neural stimulation and recording. Sensors Actuators B Chem. 132(2), 449-460 (2008)

T.L. Rose, E.M. Kelliher et al., Assesment of capacitor electrodes for intracortical neural stimulation. J. Neurosci. Methods 12(3), 181- 
193 (1985)

P.J. Rousche, D.S. Pellinen et al., Flexible polyimide-based intracortical electrode arrays with bioactive capability. IEEE Trans. Biomed. Eng. 48(3), 361-371 (2001)

Y.F. Rui, J.Q. Liu et al., Parylene-based implantable Pt-black coated flexible 3-D hemispherical microelectrode arrays for improved neural interfaces. Microsyst. Technol. 17(3), 437-442 (2011)

M. Sandison, A.S.G. Curtis et al., Effective extra-cellular recording from vertebrate neurons in culture using a new type of micro-electrode array. J. Neurosci. Methods 114(1), 63-71 (2002)

M. Shein, A. Greenbaum et al., Engineered neuronal circuits shaped and interfaced with carbon nanotube microelectrode arrays. Biomed. Microdevices 11(2), 495-501 (2009)

A. Shoval, C. Adams et al., Carbon nanotube electrodes for effective interfacing with retinal tissue. Frontiers in Neuroengineering 2 (2009)

W. S. Su, C. M. Lin et al., Patterning and growth of carbon nanotubes on a highly structured 3D substrate surface. J. Micromech. Microeng. 19(10) (2009)

H.C. Su, C.M. Lin et al., A cone-shaped 3D carbon nanotube probe for neural recording. Biosens. Bioelectron. 26(1), 220-227 (2010)
S. Takeuchi, T. Suzuki et al., 3D flexible multichannel neural probe array. J. Micromech. Microeng. 14(1), 104-107 (2004)

C. Thomsen, S. Reich, Raman scattering in carbon nanotubes in Light Scattering in Solids Ix: Novel Materials and Techniques, ed. by M. Cardona, R. Merlin, (2007), 108, 115-235

T. Y. Tsai, C. Y. Lee et al., Transfer of patterned vertically aligned carbon nanotubes onto plastic substrates for flexible electronics and field emission devices. Appl. Phys. Lett. 95(1) (2009)

J. Viventi, D.H. Kim et al., Flexible, foldable, actively multiplexed, highdensity electrode array for mapping brain activity in vivo. Nat. Neurosci. 14(12), 1599-1605 (2011)

K. Wang, H.A. Fishman et al., Neural stimulation with a carbon nanotube microelectrode array. Nano Lett. 6(9), 2043-2048 (2006)

P. Wei, R. Taylor et al., Stretchable microelectrode array using roomtemperature liquid alloy interconnects. J. Micromech. Microeng. 21(5) (2011)

B. A. Wester, R. H. Lee et al., Development and characterization of in vivo flexible electrodes compatible with large tissue displacements. J. Neural Eng 6(2) (2009) 